\title{
SOLUTIONS OF LINEAR DIFFERENTIAL SYSTEMS SATISFYING BOUNDARY CONDITIONS \\ IN THE LARGE
}

\author{
J. B. GARNER AND L. P. BURTON
}

1. Introduction. In recent years existence and uniqueness theorems have been given for differential systems where multiple-point boundary conditions are imposed. For those theorems which apply to the linear system

$$
y_{i}^{\prime}=\sum_{j=1}^{n} a_{i j}(x) y_{j}+b_{i}(x), \quad i=1, \cdots, n,
$$

the interval over which the boundary points are distributed is restricted in length. In the present paper conditions on the $a_{i j}(x)$ are given which assure a unique solution satisfying two-point and threepoint boundary conditions where these points are required only to belong to the interval, say $[a, b]$, over which the $a_{i j}(x), b_{i}(x)$ are continuous.

2. Two-point boundary conditions. For points $\alpha_{1}, \alpha_{2}, \alpha_{1}<\alpha_{2}$, of $[a, b]$ we define the following conditions over $\left[\alpha_{1}, \alpha_{2}\right]$ :

A. $a_{i j}(x), i \neq j$, is nonzero.

B. If $a_{m n}(x)>0, a_{m k}(x)$ has the same sign as $a_{k n}(x)$; if $a_{m n}(x)<0$, $a_{m k}(x)$ has the opposite sign to $a_{k n}(x) ; a_{n k}(x)$ has the same sign as $a_{k n}(x), m, k=1, \cdots, n-1, m \neq k$.

Theorem $1 .{ }^{1}$ Let the $a_{i j}(x)$ be continuous and satisfy (A), (B) for some $\alpha_{1}, \alpha_{2}$ of $[a, b]$. Then there exists a unique solution of (1) satisfying the conditions

$$
y_{k}\left(\alpha_{1}\right)=\beta_{k}, \quad y_{n}\left(\alpha_{2}\right)=\beta_{n}, \quad k=1, \cdots, n-1,
$$

where $\beta_{1}, \cdots, \beta_{n}$ are arbitrary real numbers.

Proof. Let $\left(y_{i 1}(x), \cdots, y_{i n}(x)\right), i=1, \cdots, n$, be solutions of the homogeneous system

$$
y_{i}^{\prime}=\sum_{j=1}^{n} a_{i j}(x) y_{j}
$$

satisfying

Received by the editors April 15, 1960.

1 A dual theorem may be given for $\alpha_{2}<\alpha_{1}$ if (B) is altered so that, if $a_{m n}(x)>0$, $a_{m k}(x)$ is required to have the opposite sign to $a_{k n}(x)$ and, if $a_{m n}(x)<0, a_{m k}(x)$ is required to have the same sign as $a_{k n}(x)$. 


$$
y_{i j}\left(\alpha_{1}\right)=\delta_{i j}=\left\{\begin{array}{ll}
0 & i \neq j \\
1 & i=j
\end{array} \quad i, j=1, \cdots, n .\right.
$$

Then the general solution $\left(y_{1}(x), \cdots, y_{n}(x)\right)$ of (1) is given by

$$
y_{i}(x)=c_{1} y_{1 i}(x)+c_{2} y_{2 i}(x)+\cdots+c_{n} y_{n i}(x)+y_{p i}(x),
$$

where $\left(y_{p i}(x), \cdots, y_{p n}(x)\right)$ is a particular solution of (1). Imposing the boundary conditions (2) to this system and simplifying according to (4), we obtain

$$
\begin{aligned}
c_{1} & =\beta_{1}-y_{p 1}\left(\alpha_{1}\right) \\
& : \\
c_{n-1} & =\beta_{n-1}-y_{p n-1}\left(\alpha_{1}\right) \\
c_{1} y_{1 n}\left(\alpha_{2}\right)+c_{2} y_{2 n}\left(\alpha_{2}\right)+\cdots+c_{n} y_{n n}\left(\alpha_{2}\right) & =\beta_{n}-y_{p n}\left(\alpha_{2}\right) .
\end{aligned}
$$

This system has a solution, hence (2) can be satisfied uniquely, if $y_{n n}\left(\alpha_{2}\right) \neq 0$.

Assume at least one of $y_{n 1}(x), \cdots, y_{n n}(x)$ has a zero on $\left(\alpha_{1}, \alpha_{2}\right]$. Of the $a_{m n}(x), m=1, \cdots, n-1$, let $a_{\nu^{n}}(x), p=1, \cdots, r$, be those, if any, which are positive and let $a_{\mu_{q} n}(x), q=1, \cdots, s$, be those, if any, which are negative. Then, by (4), $y_{n n}\left(\alpha_{1}\right)=1, y_{n \nu_{p}}^{\prime}\left(\alpha_{1}\right)>0$, $y_{n \mu_{q}}^{\prime}\left(\alpha_{1}\right)<0$. Hence $y_{n n}(x), y_{n n_{p}}(x)>0, y_{n \mu_{q}}(x)<0$ to the immediate right of $x=\alpha_{1}$. Now since the $y_{n j}(x)$ are continuous it is possible to let $c$ be the smallest zero of any of these functions on $\left(\alpha_{1}, \alpha_{2}\right]$. If $y_{n v_{e}}(c)$ $=0, \nu_{\varepsilon}=n$ or $1 \leqq e \leqq r$, then $y_{n v_{e}}^{\prime}(c) \leqq 0$. But, under this assumption,

$$
\begin{aligned}
y_{n v_{e}}(c)= & a_{v_{e} v_{1}}(c) y_{n v_{1}}(c)+\cdots+a_{v_{\theta} v_{e-1}}(c) y_{n v_{e}-1}(c) \\
& +a_{v_{e} v_{e+1}}(c) y_{n v_{e+1}}(c)+\cdots+a_{v_{e} v_{r}}(c) y_{n v_{r}}(c) \\
& +a_{v_{e} \mu_{1}}(c) y_{n \mu_{1}}(c)+\cdots+a_{v_{e} \mu_{e}}(c) y_{n \mu_{s}}(c) \\
& +a_{v_{e} n}(c) y_{n n}(c)
\end{aligned}
$$

is positive since, by the hypotheses and the above determined properties of $y_{n j}(x)$,

$$
a_{v_{e} v_{p}}(c) y_{n v_{p}}(c), a_{v_{e} \mu_{q}}(c) y_{n \mu_{q}}(c), a_{v_{e} n}(c) y_{n n}(c) \geqq 0,
$$

and since $y_{n 1}(x), \cdots, y_{n v_{e-1}}(x), y_{n v_{e+1}}(x), \cdots, y_{n n}(x)$ cannot all vanish at $x=c$. If $y_{n_{f}}(c)=0,1 \leqq f \leqq s$, then $y_{n \mu_{j}}^{\prime}(c) \geqq 0$. But, under this assumption, $y_{n \mu_{j}}^{\prime}(c)<0$ since

$$
a_{\mu j \mu_{q}}(c) y_{n \mu_{q}}(c), a_{\mu f v_{p}}(c) y_{n v_{p}}(c), a_{\mu f n}(c) y_{n n}(c) \leqq 0
$$

and the functions $y_{n 1}(x), \cdots, y_{n \mu_{f-1}}(x), y_{n \mu_{f+1}}(x), \cdots, y_{n n}(x)$ cannot all vanish at $x=c$. We now have a contradiction on the choice of $c$. 
Hence none of $y_{n 1}(x), \cdots, y_{n n}(x)$ vanishes on $\left(\alpha_{1}, \alpha_{2}\right]$ and the theorem follows.

COROLlary. Let the $a_{i j}(x)$ satisfy (A), (B) over $[a, b]$. Then Theorem 1 is valid without restricting the boundary points $\alpha_{1}, \alpha_{2}$ further than requiring them to belong to $[a, b]$.

Conditions are not imposed on $a_{i i}(x), i=1, \cdots, n$. If $a_{\gamma_{t} \gamma_{t}}(x) \equiv 0$ over $\left(\alpha_{1}, \alpha_{2}\right)$ for $t=1, \cdots, u(1 \leqq u \leqq n)$, the same results can be obtained with weaker restrictions than (A), (B). Of the $a_{\gamma_{t} i}(x), i=1$, $\cdots, n$, included in (B), we need require the positive functions only to be nonnegative and the negative functions to be only nonpositive with $a_{\gamma^{n}}\left(\alpha_{2}\right) \neq 0$.

3. Three-point boundary conditions. For points

$$
\alpha_{1}, \alpha_{2}, \alpha_{3}\left(\alpha_{1} \leqq \alpha_{2} \leqq \alpha_{3}\right)
$$

of $[a, b]$ we define the following conditions:

C. $a_{m m}(x)=0$ on $\left(\alpha_{1}, \alpha_{3}\right) ; a_{1 n}(x), a_{n 1}(x)>0$ on $\left[\alpha_{1}, \alpha_{3}\right]$

$$
m=2, \cdots, n-1 \text {. }
$$

D. For each $m, 2 \leqq m \leqq n-1$, either

$$
\begin{array}{rc}
a_{m 1}(x) \geqq 0 & \text { on }\left(\alpha_{1}, \alpha_{3}\right), \\
a_{m 1}\left(\alpha_{2}\right)>0 & \text { or } \\
a_{m 1}(x) \begin{cases}\geqq 0 & x \in\left(\alpha_{1}, \alpha_{2}\right], \\
\geqq 0 & x \in\left[\alpha_{2}, \alpha_{3}\right) .\end{cases}
\end{array}
$$

If (1) holds then

$$
\begin{gathered}
a_{1 m}(x)>0 \\
a_{n m}(x) \begin{cases}<0 & \text { on }\left[\alpha_{1}, \alpha_{3}\right], \\
=0 & x \in\left[\alpha_{1}, \alpha_{2}\right), \\
>0 & x \in \alpha_{2},\end{cases} \\
a_{m n}(x) \begin{cases}\leqq 0 & x \in\left(\alpha_{2}, \alpha_{3}\right], \\
\geqq 0 & x \in\left[\alpha_{1}, \alpha_{2}\right],\end{cases}
\end{gathered}
$$

If (2) holds then

$$
a_{1 m}(x) \begin{cases}<0 & x \in\left[\alpha_{1}, \alpha_{2}\right), \\ =0 & x=\alpha_{2}, \\ >0 & x \in\left(\alpha_{2}, \alpha_{3}\right],\end{cases}
$$




$$
\begin{array}{ll}
a_{n m}(x)>0 & \text { on }\left[\alpha_{1}, \alpha_{3}\right], \\
a_{m n}(x) \geqq 0 & \text { on }\left(\alpha_{1}, \alpha_{3}\right), a_{m n}\left(\alpha_{2}\right)>0 .
\end{array}
$$

E. For the case $\mathrm{D}(1)$ :

$$
\begin{array}{rlr}
a_{m k}(x)\left\{\begin{array}{lll}
\leqq 0 & x \in\left(\alpha_{1}, \alpha_{2}\right], & \\
\geqq 0 & x \in\left[\alpha_{2}, \alpha_{3}\right) & \\
a_{m k}(x) \equiv 0 & \text { on }\left(\alpha_{1}, \alpha_{3}\right) & m<k \text { if } a_{k 1}\left(\alpha_{2}\right)>0,
\end{array}\right. \\
a_{m k} \geqq 0 & \text { on }\left(\alpha_{1}, \alpha_{3}\right) & \text { if } a_{k 1}\left(\alpha_{2}\right)=0 .
\end{array}
$$

For the case $\mathrm{D}(2)$ :

$$
\begin{aligned}
& a_{m k}(x) \geqq 0 \quad \text { on }\left(\alpha_{1}, \alpha_{3}\right) \quad \text { if } a_{k 1}\left(\alpha_{2}\right)>0, \\
& a_{m k}(x)\left\{\begin{array}{ll}
\leqq 0 & x \in\left(\alpha_{1}, \alpha_{2}\right], \\
\geqq 0 & x \in\left[\alpha_{2}, \alpha_{3}\right)
\end{array} \quad m>k,\right. \\
& a_{m k}(x) \equiv 0 \quad \text { on }\left(\alpha_{1}, \alpha_{3}\right), \quad m<k \text { if } a_{k 1}\left(\alpha_{2}\right)=0 \text {. } \\
& k=2, \cdots, n-1, k \neq m \text {. }
\end{aligned}
$$

F. For each $m, 2 \leqq m \leqq n-1$, there exists a neighborhood, $\left(\alpha_{2}-\delta_{m}, \alpha_{2}+\delta_{m}\right)$, of $\alpha_{2}$ for which $a_{m 1}(x), \cdots, a_{m n}(x)$ do not all have a common zero.

LEMMA 1. Let the $a_{i j}(x)$ be continuous and satisfy (C), (D), (E), (F) for some $\alpha_{1}, \alpha_{2}, \alpha_{3}$ of $[a, b]$ and let $\left(y_{11}(x), \cdots, y_{1 n}(x)\right.$ be the solution of (3) satisfying

$$
y_{1 j}\left(\alpha_{2}\right)=\delta_{1 j}, \quad j=1, \cdots, n .
$$

Then $x=\alpha_{2}$ is an isolated zero of $y_{1 f}(x), f=2, \cdots, n$.

Proof. Of the $a_{f 1}(x), f=2, \cdots, n$, let $a_{\nu_{p} 1}(x), \nu_{1}<\nu_{2}<\cdots<\nu_{r}$ be those which are positive at $\alpha_{2}$ and let $a_{\mu_{q} 1}(x), \mu_{1}<\mu_{2}<\cdots<\mu_{s}$, be those, if any, which are zero at $\alpha_{2}$. Then, by (5), $y_{11}\left(\alpha_{2}\right)=1, y_{1 p_{p}}^{\prime}\left(\alpha_{2}\right)$ $>0, p=1, \cdots, r$. Since $y_{1 v_{p}}(x)$ is continuous and vanishes at $x=\alpha_{2}$, there exists a $\delta>0$ such that

$$
\begin{array}{ll}
y_{11}(x), y_{1 v_{p}}(x)<0 & \text { on }\left(\alpha_{2}-\delta, \alpha_{2}\right), \\
y_{11}(x), y_{1 v_{p}}(x)>0 & \text { on }\left(\alpha_{2}, \alpha_{2}+\delta\right) .
\end{array}
$$

With this and the hypotheses, we have that

$$
y_{1 \mu_{1}}^{\prime}(x)=a_{\mu_{1} 1}(x) y_{11}(x)+a_{\mu_{1} \nu_{1}}(x) y_{1 \nu_{1}}(x)+\cdots+a_{\mu_{1} \nu_{r}}(x) y_{1 v_{r}}(x)
$$

is negative over $\left(\alpha_{2}-\delta_{1}, \alpha_{2}\right)$ and positive over $\left(\alpha_{2}, \alpha_{2}+\delta_{1}\right)$, where $\delta_{1}=\min \left(\delta, \delta_{\mu_{1}}\right)$. Hence, since $y_{\mu_{1}}(x)$ vanishes at $x=\alpha_{2}$ and is continuous, $y_{1 \mu_{1}}(x)>0$ on $\left(\alpha_{2}-\delta_{1}, \alpha_{2}\right),\left(\alpha_{2}, \alpha_{2}+\delta_{1}\right)$. We now have that 


$$
\begin{aligned}
y_{1 \mu_{2}}^{\prime}(x)= & a_{\mu_{2} 1}(x) y_{11}(x)+a_{\mu_{2} \mu_{1}}(x) y_{1 \mu_{1}}(x)+a_{\mu_{2} \nu_{1}}(x) y_{1 \nu_{1}}(x) \\
& +\cdots+a_{\mu_{2} \nu_{r}}(x) y_{1 \nu_{r}}(x)
\end{aligned}
$$

is negative over $\left(\alpha_{2}-\delta_{2}, \alpha_{2}\right)$ and positive over $\left(\alpha_{2}, \alpha_{2}+\delta_{2}\right)$, where $\delta_{2}=\min \left(\delta_{1}, \delta_{\mu_{2}}\right)$. This implies that $y_{\mu_{2}}(x)>0$ on $\left(\alpha_{2}-\delta_{2}, \alpha_{2}\right)$, $\left(\alpha_{2}, \alpha_{2}+\delta_{2}\right)$. By continuing in this way we find $y_{1 \mu_{q}}(x)>0$ in a neighborhood of $x=\alpha_{2}$ for $q=1, \cdots, s$.

We have shown that each $y_{1 f}(x)$ is either positive or negative to the immediate left of $x=\alpha_{2}$ and positive to the immediate right of $x=\alpha_{2}$. Hence $\alpha_{2}$ is an isolated zero of $y_{1 f}(x), f=2, \cdots, n$.

Lemma 2. Let the $a_{i j}(x)$ be continuous and satisfy (C), (D), (E), (F) for some $\alpha_{1}, \alpha_{2}, \alpha_{3}$ of $[a, b]$ and let $\left(y_{n 1}(x), \cdots, y_{n n}(x)\right)$ be the solution of (3) satisfying

$$
y_{n j}\left(\alpha_{2}\right)=\delta_{n j}, \quad j=1, \cdots, n .
$$

Then $x=\alpha_{2}$ is an isolated zero of $y_{n e}(x), e=1, \cdots, n-1$.

Proof. By a process similar to that in the proof of Lemma 1, we find that $y_{n v_{p}}(x), p=1, \cdots, r$, is positive in a neighborhood of $x=\alpha_{2}$ and $y_{n_{q}}(x), q=1, \cdots, s$, is negative to the immediate left of $x=\alpha_{2}$, positive to the immediate right of $x=\alpha_{2}$.

TheOREM 2. Let the $a_{i j}(x)$ be continuous and satisfy (C), (D), (E), (F) for some $\alpha_{1}, \alpha_{2}, \alpha_{3}$ of $[a, b]$. Then there exists a unique solution of (1) satisfying

$$
y_{1}\left(\alpha_{1}\right)=\beta_{1}, y_{m}\left(\alpha_{2}\right)=\beta_{m}, y_{n}\left(\alpha_{3}\right)=\beta_{n}, \quad m=2, \cdots, n-1,
$$

where $\beta_{1}, \cdots, \beta_{n}$ are arbitrary real numbers.

Proof. Let $\left(y_{i 1}(x), \cdots, y_{i n}(x)\right), i=1, \cdots, n$, be solutions of $(3)$ satisfying

$$
y_{i j}\left(\alpha_{2}\right)=\delta_{i j}, \quad i, j=1, \cdots, n .
$$

Then the general solution $\left(y_{1}(x), \cdots, y_{n}(x)\right)$ of $(1)$ is given by

$$
y_{i}(x)=c_{1} y_{1 i}(x)+c_{2} y_{2 i}(x)+\cdots+c_{n} y_{n i}(x)+y_{p i}(x),
$$

where $\left(y_{p 1}(x), \cdots, y_{p n}(x)\right)$ is a particular solution (1). Imposing the boundary conditions (7) and simplifying according to (8), we obtain

$$
\begin{aligned}
c_{1} y_{11}\left(\alpha_{1}\right)+c_{2} y_{21}\left(\alpha_{1}\right)+\cdots+c_{n} y_{n 1}\left(\alpha_{1}\right) & =\beta_{1}-y_{p 1}\left(\alpha_{1}\right), \\
c_{2} & =\beta_{2}-y_{p 2}\left(\alpha_{2}\right), \\
& \vdots \\
c_{n-1} & =\beta_{n-1}-y_{p n-1}\left(\alpha_{2}\right), \\
c_{1} y_{1 n}\left(\alpha_{3}\right)+c_{2} y_{2 n}\left(\alpha_{3}\right)+\cdots+c_{n} y_{n n}\left(\alpha_{3}\right) & =\beta_{n}-y_{p n}\left(\alpha_{3}\right) .
\end{aligned}
$$


This system has a solution, hence (7) can be satisfied uniquely, if $y_{11}\left(\alpha_{1}\right) y_{n n}\left(\alpha_{3}\right)-y_{1 n}\left(\alpha_{3}\right) y_{n 1}\left(\alpha_{1}\right)$ is nonzero. We proceed to show this is the case.

Let $a_{\nu_{p} 1}(x), a_{\mu_{p} 1}(x)$ be defined as above. We first prove

$$
y_{11}(x)>0 \quad \text { on }\left[\alpha_{1}, \alpha_{2}\right) \text {. }
$$

Assume at least one of the functions $y_{11}(x), \cdots, y_{1 n}(x)$ has a zero on $\left[\alpha_{1}, \alpha_{2}\right)$. By virtue of Lemma 1 and the continuity of $y_{1 j}(x)$, $j=1, \cdots, n$, it is possible to let $c$ be the largest zero of any of these functions on $\left[\alpha_{1}, \alpha_{2}\right)$. Hence the sign of $y_{1 j}(x)$ as found in Lemma 1 holds over the interval $\left(c, \alpha_{2}\right)$.

If $y_{11}(c)=0$, then, since $y_{11}(x)>0$ on $\left(c, \alpha_{2}\right), y_{11}^{\prime}(c) \geqq 0$. But, under this assumption,

$$
\begin{aligned}
y_{11}^{\prime}(c)= & a_{1 v_{1}}(c) y_{1 v_{1}}(c)+\cdots+a_{1 v_{r}}(c) y_{1 v_{r}}(c) \\
& +a_{1 \mu_{1}}(c) y_{1 \mu_{1}}(c)+\cdots+a_{1 \mu_{s}}(c) y_{1 \mu_{s}}(c)
\end{aligned}
$$

is negative since $a_{1 v_{p}}(c) y_{1 v_{p}}(c), a_{1 \mu_{q}}(c) y_{1 \mu_{q}}(c) \leqq 0$ and the functions $y_{12}(x), \cdots, y_{1 n}(x)$ cannot all vanish at $x=c$. Hence $y_{11}(c) \neq 0$. For a similar reason, $y_{1 n}(c) \neq 0$. The function $y_{1 \mu_{q}}(x), q=1, \cdots, s$, does not vanish at $x=c$ since, from the proof of Lemma 1 , its derivative does not change sign over $\left(c, \alpha_{2}\right)$. For any $e, 1 \leqq e \leqq r-1$,

$$
\begin{aligned}
y_{1 v_{o}}^{\prime}(x)= & a_{v_{e}}(x) y_{11}(x)+a_{v_{e}: 11}(x) y_{1 \mu_{1}}(x)+\cdots \\
& +a_{v_{e} u_{o}}(x) y_{1 \mu_{e}}(x)+a_{v_{e} v_{1}}(x) y_{1 v_{1}}(x)+\cdots \\
& +a_{v_{e} v_{e}-1}(x) y_{1 v_{e}-1}(x)+a_{v_{e} v_{r}}(x) y_{1 v_{r}}(x)
\end{aligned}
$$

is positive to the immediate left of $x=\alpha_{2}$ and nonnegative over $\left(c, \alpha_{2}\right)$. Thus since $y_{1 v_{e}}\left(\alpha_{2}\right)=0, y_{1 v_{e}}(x)$ cannot vanish at $x=c$. We now have a contradiction on the choice of $c$. Hence $y_{11}(x), \cdots, y_{1 n}(x)$ do not vanish on $\left[\alpha_{1}, \alpha_{2}\right)$.

The following statements are proved in a similar manner to $(a)$ :

$$
\begin{array}{ll}
y_{n 1}(x)<0 & \text { on }\left[\alpha_{1}, \alpha_{2}\right) . \\
y_{1 n}(x)>0 & \text { on }\left(\alpha_{2}, \alpha_{3}\right] . \\
y_{n n}(x)>0 & \text { on }\left(\alpha_{2}, \alpha_{3}\right] .
\end{array}
$$

We now have $y_{11}\left(\alpha_{1}\right) y_{n n}\left(\alpha_{3}\right)-y_{1 n}\left(\alpha_{3}\right) y_{n 1}\left(\alpha_{1}\right)>0$ for $\alpha_{1}<\alpha_{2}<\alpha_{3}$. If $\alpha_{1}=\alpha_{2}<\alpha_{3}\left(\alpha_{1}<\alpha_{2}=\alpha_{3}\right)$ then the determinant in question is $y_{n n}\left(\alpha_{3}\right)>0$ $\left(y_{11}\left(\alpha_{1}\right)>0\right)$. Hence there exists a unique solution for the $c_{i}$. This in turn gives a unique solution of (1) satisfying (7).

CoRollary. Let the $a_{i j}(x)$ satisfy (C), (D), (E), (F) over the interval $\left[a, \alpha_{2}\right),\left(\alpha_{2}, b\right]$ for some $\alpha_{2} \in[a, b]$. Then. Theorem 2 is valid without 
restricting the boundary points $\alpha_{1}, \alpha_{3}$ further than requiring them to belong to $\left[a, \alpha_{2}\right],\left[\alpha_{2}, b\right]$, respectively.

Conditions are not imposed on $a_{11}(x), a_{n n}(x)$. If these functions are identically zero over $\left(\alpha_{1}, \alpha_{3}\right)$ Theorem 2 follows for weaker restrictions than (C), (D). For the $a_{1 f}(x), a_{n e}(x), f=2, \cdots, n, e=1, \cdots, n-1$, it is sufficient to require that the positive functions be nonnegative, the negative functions be nonpositive and $a_{1 n}\left(\alpha_{2}\right), a_{n 1}\left(\alpha_{2}\right)>0$.

AUbURn University

\section{A MOORE SPACE ON WHICH EVERY REAL-VALUED CONTINUOUS FUNCTION IS CONSTANT}

\section{STEVE ARMENTROUT}

F. B. Jones [2] recently gave an example of a Moore space $\Lambda_{\infty}$ in which there exists a point $x$ such that $\Lambda_{\infty}$ is not completely regular at $x$. It is easy to modify the construction used by Jones so as to obtain a Moore space $A$ in which there exist distinct points $a$ and $b$ such that for every real-valued continuous function $f$ on $A, f(a)=f(b)$. Upon applying Urysohn's process of condensation of the singularities of the space $A[4]$, in a manner similar to that used by Hewitt [1], there results a Moore space $X$ on which every real-valued continuous function is constant.

Throughout this paper, $J$ denotes the set of positive integers. A sequence is a function on $J$, and if $f$ is a sequence and $n \in J$, then $f_{n}$ denotes $f(n)$.

By a Moore space is meant a topological space $X$ whose topology has a basis consisting of sets termed regions, satisfying the following condition (axiom $1_{3}$, that is, parts 1,2 , and 3 of axiom 1 , of [3]): There exists a sequence $G$ such that (1) if $n \in J, G_{n}$ is a collection of regions covering $X$, (2) if $n \in J, G_{n+1} \subset G_{n}$, and (3) if $r$ is a region, $x \in r$, and $y \in r$, then there exists a positive integer $n$ such that if $g \in G_{n}$ and $x \in g$, then $\bar{g} \subset(r-\{x\}) \cup\{y\}$. The following characterization of a Moore space will be used in this paper: $X$ is a Moore space if and only if $X$ is a regular Hausdorff space for which there exists a sequence $G$ of open coverings of $X$ such that if $U$ is an open set and

\footnotetext{
Presented to the Society, September 3, 1959; received by the editors February 2,
} 1960. 